\title{
Synthesis and Pharmacological Evaluation of Tricyclic Carbazole Annulated with Substituted Benzothiazole-2-Amine Under Microwave Irradiation
}

\author{
Atul Sharma*1, Sanjit Kumar Das², Devender Pathak ${ }^{3}$, Gyanendra Kumar Sharma*1 \\ ${ }^{1 *}$ Department of Pharmaceutical Chemistry, Rajiv Academy for Pharmacy, Mathura, Uttar Pradesh, India. \\ ${ }^{2}$ Bengal School of Technology, Sugandha, Chuchura, West Bengal, India \\ ${ }^{3}$ Pharmacy College Safai, Uttar Pradesh University of Medical Sciences, Saifai, Etawah (UP), India
}

Received: 㠿: September 14, 2018; Published: 眥 September 25, 2018

*Corresponding author: Atul Sharma and Gyanendra Kumar Sharma, Department of Pharmaceutical Chemistry, Rajiv Academy for Pharmacy, Mathura (U.P.), India

\begin{abstract}
Tricyclic carbazole nucleus is an integral part of naturally occurring alkaloids and synthetic derivatives, possessing various potential biological activities such as anticancer, antimicrobial and antiviral. Binding mechanism of carbazole with target receptor as molecule or fused molecule exhibit potential lethal effect. In the present work, development of microwave assisted organic synthesis (MAOS) methods for synthesis of a novel series of N-Mannich bases of carbazole annulled with substituted benzothiazole-2-amine with carbazole (secondary aromatic amine) and aldehyde derivatives has been presented. All the synthesized compounds were characterized by physicochemical and spectral methods viz. ultra-violet, FT-IR, $1 \mathrm{H}-\mathrm{NMR}$, Mass and elemental analysis. The newly developed MAOS method evident the improvement in percentage yield (\% yield) and reaction time compared to conventional method. The synthesized compounds were screened for antibacterial (B. subtilis, S. aureus, E. coli and P. aeruginosa) and antifungal activities (A. niger and C. albicans) using impregnated paper-disc diffusion method. Compounds posses electronegative functional group at $\mathrm{C} 5$ and C6 position showed significant potential for antibacterial (compd. 2c and 3c) and antifungal (compd. 1b, 2b and 7b). Furthermore, in silico (PASS prediction) analysis predicts the possible mechanism of synthesized compounds could be a glycosylphosphatidylinositol phospholipase D inhibitor (antifungal action) and potassium channel blocker (antibacterial action).
\end{abstract}

Keywords: Microwave assisted organic synthesis (MAOS); Carbazole; Benzothiazole; Antimicrobial activity; N-Mannich bases

\section{Introduction}

Tricyclic carbazole nucleus is an integral part of naturally occurring alkaloids and synthetic derivatives, possessing various biological activities such as antiviral [1] antibacterial [2] antimicrobial [3] anti-inflammatory [4] antifungal, antiprotozoological [5] antioxidant [6] antidiarrhoeal [7] anticancer [8] etc. The derivatization of carbazole derivatives, especially with fused heterocyclic compounds has attained a significant attention in search of newer antimicrobial molecules and anticancer with an enhanced pharmacological activity using bio-isosteric replacements $[9,10]$. The development of newer compounds with enhanced activity of carbazole nucleus is constantly under investigation due to their high affinity towards DNA.

This high affinity is based on their intercalation property in the minor groove of DNA, which is attributed to the secondary amine (>NH) group of carbazole moiety [11,12] In recent research, the development of N-Mannich bases of heterocyclic moieties significantly become important due to the rendering of molecule aqueous solubility when transferred to the ammonium salt [13]. The N-Mannich bases are the compounds with powerful bond between carbon atoms having wide application to prepare diverse amino alkyl derivatives involving the condensation of active hydrogen containing molecules with aldehyde and primary or secondary amine [14]. The existing literature suggested the synthesis of N-Mannich bases derived from different heterocyclic moieties i.e. carbazole, triazole, benzimidazole possessing wide spectrum pharmacological activities [15-18].

Recently, the integration of microwave assisted organic synthesis (MAOS) in drug discovery has become an invaluable technique. Indeed, the use of microwave irradiation is simplified and improves the classical organic reactions which lead to the higher yields, cleaner reaction and shorter time of reaction [19]. Thus, based on the expectation of the wide application of carbazole nucleus and MAOS reaction, the present work employs the development of MAOS methods for the synthesis of new series of N-Mannich bases of carbazole. The developed MAOS method 
was compared with conventional adopted method including the percentage yield (\% yield) and reaction time. MAOS reactions showed the better \% yield and less reaction time. The synthesized compounds were experimentally screened for antibacterial activity against gram positive ( $S$. aureus and B. subtilis) and gram negative (P. aeruginosa and $E$. coli) bacteria while antifungal activity was screened against $A$. niger and C. albicans. Later on, the in-silico predicted biological activities were compared with experimentally performed activities.

\section{Experimental}

\section{Materials and Reagents}

Carbazole was purchased from M/s Sigma Aldrich (India). All other chemicals like ammonium thiocyanate, glacial acetic acid, toluene, methanol, ethanol, n-octanol, bromine, concentrated hydrochloric acid (conc. $\mathrm{HCl}$ ), formaldehyde, aniline derivatives and $m$-hydroxybenzaldehyde used were of analytical grade. All the solvents used in the synthesis and chromatographic analysis were distilled before use and store properly when not in use.

\section{General Procedure for Synthesis of Substituted Benzo[D] Thiazol-2-Amine (1a-7a): Conventional Method}

Equimolar quantity of substituted anilines $(20 \mathrm{mmol})$ and ammonium thiocyanate $(20 \mathrm{mmol})$ were dissolved in ethanol $(40 \mathrm{~mL})$ followed by addition of $2.0 \mathrm{~mL}$ of conc. $\mathrm{HCl}$ in a round bottom flask. To the above solution, separately prepared bromine in glacial acetic acid (50 mmol) mixture was added under ice-cold condition and refluxed. TLC was performed to investigate the completion of the reactions. On the basis of TLC performance, the reaction mixture was cooled overnight at $4^{\circ} \mathrm{C}$ and filtered. The obtained product was dried and recrystallized from absolute ethanol.

\section{Microwave Assisted Organic Synthesis (MAOS)}

Similarly, the equimolar quantity of all reagents were taken and added in iodine flask (quantities explained in conventional method). The reaction mixture was irradiated in microwave at $750 \mathrm{~W}$ with intermittent cooling and mixing after 1 minute (unless the condition was not optimized). Completion of the reaction was monitored by TLC after every 1 minute in toluene: methanol (7:3). Based on TLC performance, the reaction mixture was carried out and cooled in ice cold conditions. Crystalline product was filtered out and dried. The dried product was again recrystallized from absolute ethanol.

General procedure for the synthesis of N-Mannich bases of $\mathrm{N}$-[(9H-carbazol-9-yl) methyl] substituted benzo[d] thiazol-2-amine (1b-7b) and 3-[(4-Chlorobenzo[d]thiazol-2-yl amino) (9H-carbazol-9-yl) methyl] phenol (1c7c): Conventional Method

Equimolar quantity of carbazole and substituted benzo[d]thiazol-2-amine (1a-7a) (5mmol) precursor were dissolved in methanol $(60 \mathrm{~mL})$ in a round bottom flask. The equimolar quantity of aldehyde (formaldehyde or $m$-hydroxybenzaldehyde) ( $5 \mathrm{mmol}$ ) was added slowly with continuous stirring followed by drop by drop addition of $1.5 \mathrm{~mL}$ of conc. HCl. The obtained mixture was refluxed. Completion of reaction was confirmed by TLC after each $30 \mathrm{~min}$ in toulene: methanol (7:3). On the basis of TLC performance, the reaction mixture was cooled overnight in ice cold condition and filtered out. The obtained products was dried and recrystallized from absolute ethanol.

\section{MAOS Method}

Similarly, the equimolar quantity of reagents (carbazole and substituted benzothiazole precursor) was dissolved in methanol $(60 \mathrm{~mL})$ in an iodine flask. The equimolar quantity of aldehyde (5.0 mmol) was added with continuous stirring followed by drop by drop addition of $1.5 \mathrm{~mL}$ of conc. HCl. Under optimized conditions, the reaction mixture was irradiated in microwave at 750 Watt with intermittent cooling and mixing. The completion of the reaction was monitored using TLC performance in toluene: methanol (7:3) at every minute. Thus, on the basis of TLC results, the reaction mixture was taken out and cooled in ice cold condition. The crystalline product was filtered, dried and recrystallized from absolute ethanol.

\section{Characterization}

Physicochemical Analysis: The melting point ranges of the newly synthesized compounds were determined by open capillary method, using the melting point apparatus and were uncorrected. The solubility was evaluated in various solvents (distilled water and organic solvents) to optimize the condition for further spectral analysis. Thin layer chromatography (TLC) was carried out on silica gel-G coated plates to monitor the reaction conditions and to determine the purity of the products. The mobile phase was selected according to the polarity of the compounds and a mixture of toulene to methanol (7:3) was optimized in further experimentation. Visualization was achieved by exposure to iodine vapors and UV light (254 nm, short wavelength) for calculation of Rf values. The partition coefficient (octanol/phosphate buffer pH 7.4) of synthesized compounds was determined at room temperature by the flask shake method. Theoretical $\log P$ for synthesized compounds was compared with the experimental logP data. Structure and molecular weight of synthesized compounds were elucidated on the basis of spectral analysis.

Spectroscopical Characterization: UV absorption measurements were performed to determine the absorption maxima ( $\lambda$ max) in absolute ethanol on UV-Visible Spectrophotometer Pharma spec-1700 (M/s SHIMADZU). Fourier transform infrared spectrum (FTIR) was recorded on Fourier Transform Infrared Spectrophotometer (FTIR-8400S, M/s SHIMADZU, Japan) using the $\mathrm{KBr}$ disk method. 1H NMR (300 MHz) and D2O exchange spectrum were recorded on Bruker AvIII HD-300 MHz FT, Switzerland. All the spectra were recorded in DMSO-d6 using TMS as internal standard. The chemical shifts are reported in parts per million (ppm). Mass spectra of newly synthesized compounds were recorded on Waters UPLC-TQD Mass spectrometer model spectrophotometer and elemental analysis was carried out using Euro-E 3000 elemental analyzer.

Software Analysis: Chem draw ultra and Chem Draw 3D software were used to draw the chemical structure and to determine the IUPAC nomenclature, molecular weight and molecular formula of newly synthesized compounds. For structural elucidation, theo- 
retical obtained elemental fragmentation was compared with the experimentally obtained results.

\section{Data Set Modeling}

Schrodinger's Maestro 3D module was used for data set modeling (www.schrodinger.com). The partial atomic charges were assigned using the Gasteiger method. Energy optimization cycles were used for the initial geometry optimization of the molecules. Annealing dynamics was used for conformational scanning with user defined attributes: required temperature-450 K, dynamic time step- 0.01 ps, and iteration-10000, at NVE (constant number of moles, volume and energy). Simulated annealing dynamics were followed by energy minimization using MMF94 force field and molecules were minimized to the high convergence using 1000 iterations or more as required. 2.5. Pharmacological Screening The newly synthesized compounds $(1 \mathrm{~b}-7 \mathrm{~b}$ and $1 \mathrm{c}-7 \mathrm{c})$ were screened for their in vitro antibacterial activity against Gram positive ( $B$. subtilis and S. aureus) and Gram negative (E. coli and P. aeruginosa) bacteria using paper disc diffusion method [20] In brief, the test compounds were dissolved in DMSO at concentration 25, 50 and $100 \mu \mathrm{g} / \mathrm{mL}$ and paper disc were impregnated. For control, the disc impregnated with DMSO was used.

The microorganism culture was spread over nutrient agar media in petri dishes and each plate area was divided into 5 equal portions. Three discs of test sample, one disc of reference drug ci- profloxacin $(50 \mu \mathrm{g} / \mathrm{mL})$ and one control were placed in different sections. Thus, the plates were incubated at $35^{\circ} \mathrm{C}$ for next $24 \mathrm{hrs}$ to determine the antibacterial activities. After incubation, the zones of inhibition were calculated from triplicate measurements. Similarly, the in vitro antifungal screening was carried out against two fungal strains (A. niger and C. albicans). A spore suspension in normal saline was prepared from the culture of the test fungi in sabouraud's broth media. Fluconazole $(50 \mu \mathrm{g} / \mathrm{mL})$ was used as standard drug and disc impregnated with the solvent (DMSO) used as negative control. The three different concentration test compounds, i.e. 50, 100 and $200 \mu \mathrm{g} / \mathrm{mL}$ in DMSO were used for antifungal measurements. The petri plates inoculated with fungal cultures with standard and test compounds were incubated at $25^{\circ} \mathrm{C}$ for $48 \mathrm{hrs}$. Antifungal activity was determined by measuring and calculating the diameter of zone of inhibition in triplicate.

\section{Result and Discussion}

In the twostep reaction for the synthesis of N-Mannich bases of carbazole, initially the synthesis of substituted benzothiazole precursors (1a-7a) was accomplished by the reaction of ammonium thiocyanate followed using bromine cyclization mechanism Figure 1. Thus, based on the synthesis of substituted benzothiazole attention turns towards the synthesis of N-Mannich bases. Further, the synthesized compounds were evaluated for in vitro antimicrobial activities.

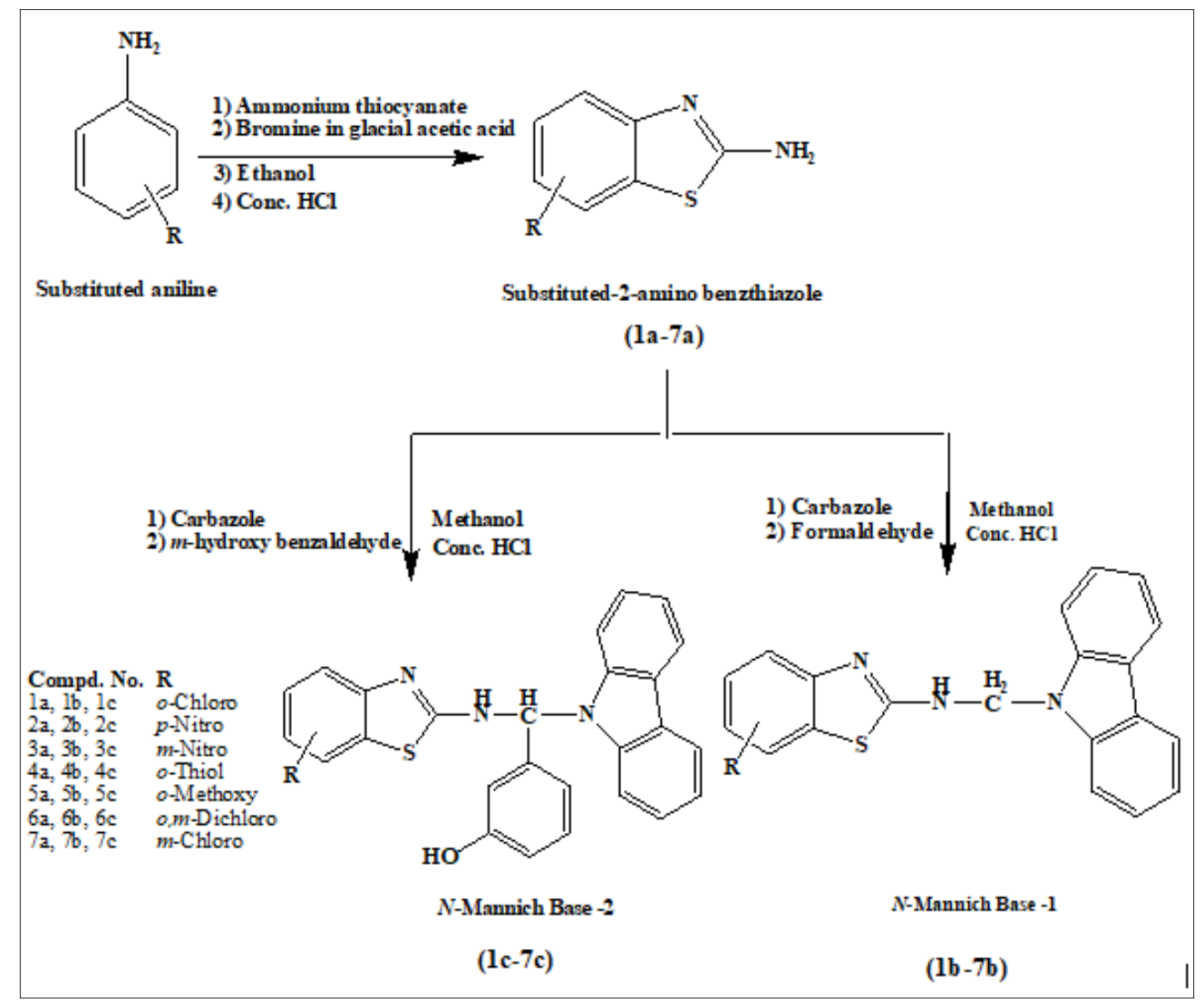

Figure 1: Synthesis of benzothiazole precursors and N-Mannich bases of carbazole. 


\section{Synthesis of Substituted Benzothiazole Precursors (1a-} 7a)

Substituted benzothiazoles (1a-7a) were synthesized from substituted anilines and ammonium thiocyanate using bromine cyclization mechanism. On the basis of TLC, the substituted benzothiazole precursors were characterized and used in the next step. The reaction performance of conventional and MAOS method was compared. Encouragingly, the developed MAOS shows better \% yield and less reaction time to accomplished reaction in compar- ison to the conventional reflux method as shown in Figure 2. The obtained results are summarized in supplementary table ST 1. 3.2. Synthesis of N-[(9H-carbazol-9-yl) methyl]-halobenzo[d]thiazol-2amine (1b-7b) and 3-[(Halobenzo[d]thiazol-2-ylamino) (9H-carbazol-9-yl) methyl] phenol (1c-7c) N-Mannich bases of carbazole were synthesized by the reaction of carbazole, aldehydes and substituted benzothiazole derivatives. Both the conventional as well as developed MAOS methods were adopted for the synthesis of N-Mannich bases.

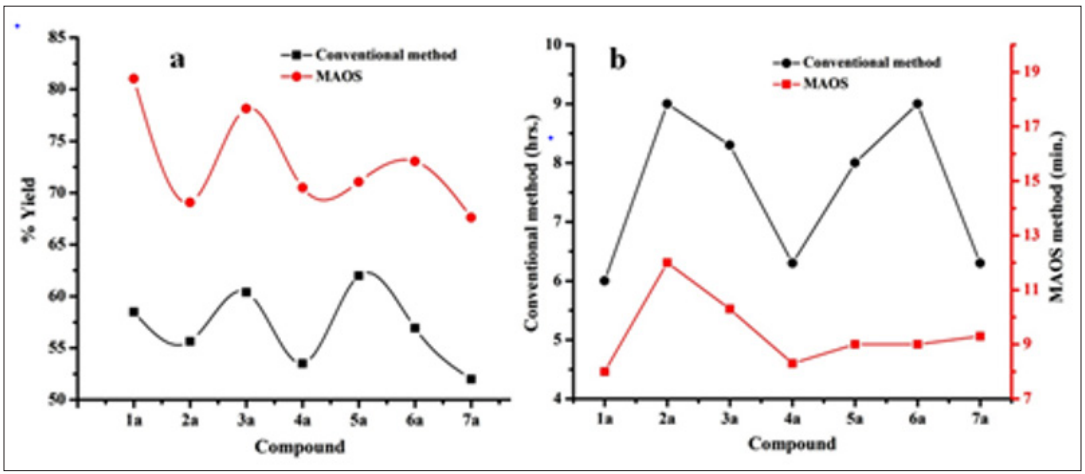

Figure 2: Comparative results of conventional and MAOS method substituted benzothiazole-2-amine (1a-7a) showing (a) \% yield and (b) reaction time.

All newly synthesized compounds were physicochemical (Rf, $\log$ P) and spectroscopically characterized using FT-IR, 1H-NMR, Mass and elemental analysis. The $1 \mathrm{H}-\mathrm{NMR}$ spectra showed characteristic peak in DMSO-d6 at 5.643-5.698 $\delta$ (singlet 2H) -CH2 methylene bridge, $10.438-10.644 \delta$ (singlet $1 \mathrm{H}$ ) of $-\mathrm{NH}$ in 1b-7b. Similarly, the NMR peak of 5.09-6.120 $\delta$ (singlet 1H) -CHC6H5, 9.043-9.103 $\delta$ (br. singlet $1 \mathrm{H}$ ) of $-\mathrm{OH}, 10.892-10.993 \delta$ (singlet $1 \mathrm{H}$ ) of $-\mathrm{NH}$ in 1c-7c confirm the formation of mannich bases. The FTIR spectrum characteristic peaks were obtained at $(\mathrm{KBr}) \mathrm{v} \mathrm{cm} 1$ at approximately $3409,3047,2923,1649,1487$ and 1058. The spectral analysis results confirm the formations of compounds. The obtained results are summarized in Tables 1 \& 2. Experimentally obtained elemental results were in good agreements to the theoretical obtained valve. The developed MAOS showed the significant improvement in \% yield and reaction time as shown in Table 2.

Table 1: Physicochemical and elemental data of newly prepared compounds as N-Mannich's bases 1b-7b and 1c-7c.

\begin{tabular}{|c|c|c|c|c|c|c|c|c|}
\hline \multirow{2}{*}{ Comp. No. } & \multirow{2}{*}{ Rf } & \multirow{2}{*}{$\log P$} & \multirow{2}{*}{ Melting point } & \multirow{2}{*}{ Molecular formula $\left(M_{w}\right)$} & \multicolumn{4}{|c|}{ Found/calcd. (\%) } \\
\hline & & & & & C & H & $\mathbf{N}$ & $S$ \\
\hline \multirow{2}{*}{$1 b$} & \multirow{2}{*}{0.59} & \multirow{2}{*}{6.18} & \multirow{2}{*}{$215-216^{\circ} \mathrm{C}$} & $\mathrm{C}_{20} \mathrm{H}_{1} 4 \mathrm{ClN}_{3} \mathrm{~S}$ & 66.02 & 3.88 & 11.55 & 8.81 \\
\hline & & & & $(364)$ & 66.05 & 3.91 & 11.56 & 8.8 \\
\hline \multirow{2}{*}{$2 b$} & \multirow{2}{*}{0.63} & \multirow{2}{*}{4.78} & \multirow{2}{*}{$201-202^{\circ} \mathrm{C}$} & $\mathrm{C}_{20} \mathrm{H}_{14} \mathrm{~N}_{4} \mathrm{O}_{2} \mathrm{~S}$ & 64.16 & 3.77 & 14.96 & 8.56 \\
\hline & & & & (374) & 64.13 & 3.74 & 14.94 & 8.55 \\
\hline \multirow{2}{*}{$3 b$} & \multirow{2}{*}{0.84} & \multirow{2}{*}{4.73} & \multirow{2}{*}{$191-192^{\circ} \mathrm{C}$} & $\mathrm{C}_{20} \mathrm{H}_{14} \mathrm{~N}_{4} \mathrm{O}_{2} \mathrm{~S}$ & 64.16 & 3.77 & 14.96 & 8.56 \\
\hline & & & & $(374)$ & 64.13 & 3.74 & 14.92 & 8.58 \\
\hline \multirow{2}{*}{$4 b$} & \multirow{2}{*}{0.54} & \multirow{2}{*}{5.81} & \multirow{2}{*}{$219-220^{\circ} \mathrm{C}$} & $\mathrm{C}_{20} \mathrm{H}_{15} \mathrm{~N}_{3} \mathrm{~S}_{2}$ & 66.45 & 4.18 & 11.62 & 17.74 \\
\hline & & & & $(361)$ & 66.48 & 4.14 & 11.65 & 17.72 \\
\hline \multirow{2}{*}{$5 b$} & \multirow{2}{*}{0.78} & \multirow{2}{*}{5.5} & \multirow{2}{*}{$182-183^{\circ} \mathrm{C}$} & $\mathrm{C}_{21} \mathrm{H}_{17} \mathrm{~N}_{3} \mathrm{OS}$ & 70.17 & 4.77 & 11.69 & 8.92 \\
\hline & & & & (359) & 70.19 & 4.74 & 11.68 & 8.96 \\
\hline \multirow{2}{*}{$6 b$} & \multirow{2}{*}{0.7} & \multirow{2}{*}{6.74} & \multirow{2}{*}{$211-212^{\circ} \mathrm{C}$} & $\mathrm{C}_{20} \mathrm{H}_{13} \mathrm{Cl}_{2} \mathrm{~N}_{3} \mathrm{~S}$ & 60.31 & 3.29 & 10.55 & 8.05 \\
\hline & & & & (397) & 60.33 & 3.32 & 11.52 & 8.03 \\
\hline \multirow{2}{*}{$7 b$} & \multirow{2}{*}{0.73} & \multirow{2}{*}{6.18} & \multirow{2}{*}{$191-192^{\circ} \mathrm{C}$} & $\mathrm{C}_{20} \mathrm{H}_{14} \mathrm{ClN}_{3} \mathrm{~S}$ & 66.02 & 3.88 & 11.55 & 8.81 \\
\hline & & & & $(364)$ & 65.98 & 3.9 & 11.53 & 8.8 \\
\hline \multirow{2}{*}{$1 c$} & \multirow{2}{*}{0.76} & 7.69 & $189-190^{\circ} \mathrm{C}$ & $\mathrm{C}_{26} \mathrm{H}_{18} \mathrm{ClN}_{3} \mathrm{OS}$ & 68.49 & 3.98 & 9.22 & 7.03 \\
\hline & & & & $(456)$ & 68.48 & 3.94 & 9.21 & 7.06 \\
\hline
\end{tabular}




\begin{tabular}{|c|c|c|c|c|c|c|c|c|}
\hline $2 c$ & 0.55 & 5.31 & $190-191^{\circ} \mathrm{C}$ & $\begin{array}{c}\mathrm{C}_{26} \mathrm{H}_{18} \mathrm{~N}_{4} \mathrm{O}_{3} \mathrm{~S} \\
(467)\end{array}$ & $\begin{array}{l}66.94 \\
66.93\end{array}$ & $\begin{array}{l}3.89 \\
3.84\end{array}$ & $\begin{array}{l}12.01 \\
12.02\end{array}$ & $\begin{array}{l}6.87 \\
6.85\end{array}$ \\
\hline $3 c$ & 0.81 & 5.61 & $177-178^{\circ} \mathrm{C}$ & $\begin{array}{c}\mathrm{C}_{26} \mathrm{H}_{18} \mathrm{~N}_{4} \mathrm{O}_{3} \mathrm{~S} \\
(467)\end{array}$ & $\begin{array}{l}66.94 \\
66.91\end{array}$ & $\begin{array}{l}3.89 \\
3.88\end{array}$ & $\begin{array}{l}12.01 \\
12.04\end{array}$ & $\begin{array}{l}6.87 \\
6.88\end{array}$ \\
\hline $4 c$ & 0.54 & 7.32 & $166-167^{\circ} \mathrm{C}$ & $\begin{array}{c}\mathrm{C}_{26} \mathrm{H}_{19} \mathrm{~N}_{3} \mathrm{OS}_{2} \\
(454)\end{array}$ & $\begin{array}{l}68.85 \\
68.83\end{array}$ & $\begin{array}{l}4.22 \\
4.24\end{array}$ & $\begin{array}{l}9.26 \\
9.24\end{array}$ & $\begin{array}{l}14.14 \\
14.12\end{array}$ \\
\hline $5 c$ & 0.75 & 7.01 & $172-173^{\circ} \mathrm{C}$ & $\begin{array}{c}\mathrm{C}_{27} \mathrm{H}_{21} \mathrm{~N}_{3} \mathrm{O}_{2} \mathrm{~S} \\
(452)\end{array}$ & $\begin{array}{l}71.82 \\
71.85\end{array}$ & $\begin{array}{l}4.69 \\
4.67\end{array}$ & $\begin{array}{l}9.31 \\
9.32\end{array}$ & $\begin{array}{c}7.1 \\
7.11\end{array}$ \\
\hline $6 c$ & 0.58 & 8.25 & $181-182^{\circ} \mathrm{C}$ & $\begin{array}{c}\mathrm{C}_{26} \mathrm{H}_{17} \mathrm{Cl}_{2} \mathrm{~N}_{3} \mathrm{OS} \\
(490)\end{array}$ & $\begin{array}{l}63.68 \\
63.69\end{array}$ & $\begin{array}{l}3.49 \\
3.46\end{array}$ & $\begin{array}{l}8.57 \\
8.53\end{array}$ & $\begin{array}{l}6.54 \\
6.57\end{array}$ \\
\hline $7 \mathrm{c}$ & 0.63 & 7.69 & $168-169^{\circ} \mathrm{C}$ & $\begin{array}{c}\mathrm{C}_{26} \mathrm{H}_{18} \mathrm{Cl} \mathrm{N}_{3} \mathrm{OS} \\
(456)\end{array}$ & $\begin{array}{l}68.49 \\
68.51\end{array}$ & $\begin{array}{l}3.98 \\
3.99\end{array}$ & $\begin{array}{l}9.22 \\
9.24\end{array}$ & $\begin{array}{l}7.03 \\
7.04\end{array}$ \\
\hline
\end{tabular}

Table 2: Spectral analysis data for synthesized compounds $1 \mathrm{~b}-7 \mathrm{~b}$ and 1c-7c.

\begin{tabular}{|c|c|c|c|}
\hline Comp. No. & FT-IR (v, cm-1) & 1H NMR signals ( $\delta, \mathrm{ppm})$ & MS (m/z) \\
\hline $1 b$ & 3409, 3047, 2923, 1649, 1487, 1058. & $\begin{array}{c}\left.\text { 5.643 (2H, s, } \mathrm{CH}_{2}\right), 7.129-7.174(2 \mathrm{H}, \mathrm{t}, \mathrm{Ar}-\mathrm{H}), 7.359-7.401(2 \mathrm{H}, \mathrm{t}, \mathrm{Ar}-\mathrm{H}), 7.456-7.473 \\
(2 \mathrm{H}, \mathrm{d}, \mathrm{Ar}-\mathrm{H}), 7.478-7.494(1 \mathrm{H}, \mathrm{t}, \mathrm{Ar}-\mathrm{H}), 7.543-7.559(1 \mathrm{H}, \mathrm{d}, \mathrm{Ar}-\mathrm{H}), 7.729-7.758 \\
(2 \mathrm{H}, \mathrm{d}, \mathrm{Ar}-\mathrm{H}), 7.989-8.002(1 \mathrm{H}, \mathrm{d}, \mathrm{Ar}-\mathrm{H}), 10.479\left(1 \mathrm{H}, \mathrm{s},-\mathrm{NH}, \mathrm{D}_{2} \mathrm{O} \text { exchangeable }\right)\end{array}$ & 363 \\
\hline $2 \mathrm{~b}$ & 3413, 3051, 2925, 1652, 1504, 1489. & $\begin{array}{c}5.680\left(2 \mathrm{H}, \mathrm{s}, \mathrm{CH}_{2}\right), 7.134-7.183(2 \mathrm{H}, \mathrm{t}, \mathrm{Ar}-\mathrm{H}), 7.362-7.412(2 \mathrm{H}, \mathrm{t}, \mathrm{Ar}-\mathrm{H}), 7.483- \\
7.513(2 \mathrm{H}, \mathrm{d}, \mathrm{Ar}-\mathrm{H}), 7.724-7.751(2 \mathrm{H}, \mathrm{d}, \mathrm{Ar}-\mathrm{H}), 8.236-8.282(2 \mathrm{H}, \mathrm{d}, \mathrm{Ar}-\mathrm{H}), 8.385 \\
(1 \mathrm{H}, \mathrm{s}, \mathrm{Ar}-\mathrm{H}), 10.542\left(1 \mathrm{H}, \mathrm{s},-\mathrm{NH}, \mathrm{D}_{2} \mathrm{O} \text { exchangeable }\right)\end{array}$ & 374 \\
\hline $3 b$ & $\begin{array}{l}3417,3047,2932,2954,1647,1506 \\
1492 .\end{array}$ & $\begin{array}{c}\left.\text { 5.698 (2H, s, } \mathrm{CH}_{2}\right), 7.144-7.178(2 \mathrm{H}, \mathrm{t}, \mathrm{Ar}-\mathrm{H}), 7.362-7.418(2 \mathrm{H}, \mathrm{t}, \mathrm{Ar}-\mathrm{H}), 7.488- \\
7.512(2 \mathrm{H}, \mathrm{d}, \mathrm{Ar}-\mathrm{H}), 7.734-7.761(2 \mathrm{H}, \mathrm{d}, \mathrm{Ar}-\mathrm{H}), 8.256-8.311(2 \mathrm{H}, \mathrm{d}, \mathrm{Ar}-\mathrm{H}), 8.415 \\
(1 \mathrm{H}, \mathrm{s}, \mathrm{Ar}-\mathrm{H}), 10.602\left(1 \mathrm{H}, \mathrm{s},-\mathrm{NH}, \mathrm{D}_{2} \mathrm{O} \text { exchangeable }\right)\end{array}$ & 374 \\
\hline $4 \mathrm{~b}$ & 3413, 3049, 2941, 2609, 1654, 1484. & $\begin{array}{c}\text { 3.493 (1H, s, - } \mathrm{SH}, \mathrm{D}_{2} \mathrm{O} \text { exchangeable), } 5.644\left(2 \mathrm{H}, \mathrm{s}, \mathrm{CH}_{2}\right), 7.141-7.179(2 \mathrm{H}, \mathrm{t}, \mathrm{Ar}-\mathrm{H}) \\
\text { 7.368-7.409 }(3 \mathrm{H}, \mathrm{t}, \mathrm{Ar}-\mathrm{H}), 7.479-7.515(2 \mathrm{H}, \mathrm{t}, \mathrm{Ar}-\mathrm{H}), 7.594-7.609(1 \mathrm{H}, \mathrm{d}, \mathrm{Ar}-\mathrm{H}), \\
\text { 7.719-7.739 (2H, d, Ar-H), 7.991-8.006 }(1 \mathrm{H}, \mathrm{d}, \mathrm{Ar}-\mathrm{H}), 10.644\left(1 \mathrm{H}, \mathrm{s},-\mathrm{NH}, \mathrm{D}_{2} \mathrm{O}\right. \\
\text { exchangeable) }\end{array}$ & 361 \\
\hline $5 b$ & $\begin{array}{c}3421,3051,2951,2942,1649,1491 \\
1242\end{array}$ & $\begin{array}{c}3.740\left(3 \mathrm{H}, \mathrm{s}, \mathrm{OCH}_{3}\right), 5.654(2 \mathrm{H}, \mathrm{s}, \mathrm{CH} 2), 6.994-7.064(1 \mathrm{H}, \mathrm{d}, \mathrm{Ar}-\mathrm{H}), 7.134-7.177 \\
(2 \mathrm{H}, \mathrm{t}, \mathrm{Ar}-\mathrm{H}), 7.368-7.404(2 \mathrm{H}, \mathrm{t}, \mathrm{Ar}-\mathrm{H}), 7.435-7.461(1 \mathrm{H}, \mathrm{t}, \mathrm{Ar}-\mathrm{H}), 7.475-7.501(2 \mathrm{H}, \\
\text { t, Ar-H), 7.677-7.697 (1H, d, Ar-H), 7.728-7.746 (2H, d, Ar-H), 10.645 (1H, s, -NH, } \\
\left.\mathrm{D}_{2} \mathrm{O} \text { exchangeable }\right)\end{array}$ & 359 \\
\hline $6 b$ & $3423,3045,2923,1651,1486,1054$ & $\begin{array}{c}5.644\left(2 \mathrm{H}, \mathrm{s}, \mathrm{CH}_{2}\right), 7.130-7.179(2 \mathrm{H}, \mathrm{t}, \mathrm{Ar}-\mathrm{H}), 7.361-7.402(2 \mathrm{H}, \mathrm{t}, \mathrm{Ar}-\mathrm{H}), 7.457- \\
7.478(2 \mathrm{H}, \mathrm{t}, \mathrm{Ar}-\mathrm{H}), 7.496-7.509(1 \mathrm{H}, \mathrm{t}, \mathrm{Ar}-\mathrm{H}), 7.729-7.755(2 \mathrm{H}, \mathrm{d}, \mathrm{Ar}-\mathrm{H}), 7.978- \\
7.994(1 \mathrm{H}, \mathrm{d}, \mathrm{Ar}-\mathrm{H}), 10.478\left(1 \mathrm{H}, \mathrm{s}, \mathrm{s}-\mathrm{NH}, \mathrm{D}_{2} \mathrm{O} \text { exchangeable }\right)\end{array}$ & 397 \\
\hline $7 \mathrm{~b}$ & $3419,3049,2926,1643,1486,1054$ & $\begin{array}{c}5.698\left(2 \mathrm{H}, \mathrm{s}, \mathrm{CH}_{2}\right), 7.134-7.174(2 \mathrm{H}, \mathrm{t}, \mathrm{Ar}-\mathrm{H}), 7.356-7.401(2 \mathrm{H}, \mathrm{t}, \mathrm{Ar}-\mathrm{H}), 7.478- \\
7.498(2 \mathrm{H}, \mathrm{A}, \mathrm{Ar}-\mathrm{H}), 7.553-7.569(1 \mathrm{H}, \mathrm{d}, \mathrm{Ar}-\mathrm{H}), 7.728-7.756(2 \mathrm{H}, \mathrm{d}, \mathrm{Ar}-\mathrm{H}), 7.989- \\
8.006(1 \mathrm{H}, \mathrm{d}, \mathrm{Ar}-\mathrm{H}), 8.243(1 \mathrm{H}, \mathrm{s}, \mathrm{Ar}-\mathrm{H}), 10.438\left(1 \mathrm{H}, \mathrm{s},-\mathrm{NH}, \mathrm{D}_{2} \mathrm{O} \text { exchangeable). }\right.\end{array}$ & 363 \\
\hline $1 \mathrm{c}$ & $\begin{array}{l}3622,3422,3044,2928,2954,1649 \\
1487,1232,1061\end{array}$ & $\begin{array}{c}6.109\left(1 \mathrm{H}, \mathrm{s},-\mathrm{CHC}_{6} \mathrm{H}_{5}\right), 7.009(1 \mathrm{H}, \mathrm{s}, \mathrm{Ar}-\mathrm{H}), 7.111-7.128(2 \mathrm{H}, \mathrm{d}, \mathrm{Ar}-\mathrm{H}), 7.130-7.176 \\
(3 \mathrm{H}, \mathrm{t}, \mathrm{Ar}-\mathrm{H}), 7.356-7.400(1 \mathrm{H}, \mathrm{t}, \mathrm{Ar}-\mathrm{H}), 7.409-7.423(1 \mathrm{H}, \mathrm{t}, \mathrm{Ar}-\mathrm{H}), 7.454-7.473(2 \mathrm{H}, \\
\mathrm{d}, \mathrm{Ar}-\mathrm{H}), 7.479-7.496(1 \mathrm{H}, \mathrm{t}, \mathrm{Ar}-\mathrm{H}), 7.541-7.558(1 \mathrm{H}, \mathrm{d}, \mathrm{Ar}-\mathrm{H}), 7.729-7.761(1 \mathrm{H}, \mathrm{d}, \\
\mathrm{Ar}-\mathrm{H}), 7.794-7.806(1 \mathrm{H}, \mathrm{d}, \mathrm{Ar}-\mathrm{H}), 7.988-8.002(1 \mathrm{H}, \mathrm{d}, \mathrm{Ar}-\mathrm{H}), 9.073(1 \mathrm{H}, \mathrm{br} \mathrm{s}, \mathrm{OH} \\
\text { D20 exchangeable), } 10.943\left(1 \mathrm{H}, \mathrm{s},-\mathrm{NH}, \mathrm{D}_{2} \mathrm{O} \text { exchangeable }\right)\end{array}$ & 455 \\
\hline $2 c$ & $\begin{array}{l}3631,3415,3051,2925,1648,1500 \\
1482,1240 .\end{array}$ & 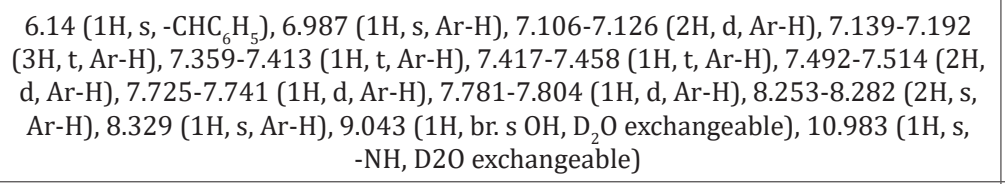 & 466 \\
\hline $3 c$ & $\begin{array}{l}3634,3419,3054,2930,1649,1498 \\
1485,1253 .\end{array}$ & $\begin{array}{c}6.113\left(1 \mathrm{H}, \mathrm{s},-\mathrm{CHC}_{6} \mathrm{H}_{5}\right), 6.988(1 \mathrm{H}, \mathrm{s}, \mathrm{Ar}-\mathrm{H}), 7.104-7.124(2 \mathrm{H}, \mathrm{d}, \mathrm{Ar}-\mathrm{H}), 7.136-7.194 \\
(3 \mathrm{H}, \mathrm{t}, \mathrm{Ar}-\mathrm{H}), 7.361-7.409(1 \mathrm{H}, \mathrm{t}, \mathrm{Ar}-\mathrm{H}), 7.413-7.452(1 \mathrm{H}, \mathrm{t}, \mathrm{Ar}-\mathrm{H}), 7.490-7.514(2 \mathrm{H}, \\
\text { d, Ar-H), 7.739-7.756 (1H, d, Ar-H), 7.794-7.805 (1H, d, Ar-H), 8.258-8.304 (2H, s, } \\
\text { Ar-H), 8.415 (1H, s, Ar-H), 9.103 (1H, br. s. OH, } \mathrm{D}_{2} \mathrm{O} \text { exchangeable), } 10.993(1 \mathrm{H}, \mathrm{s}, \\
\left.\text {-NH, } \mathrm{D}_{2} \mathrm{O} \text { exchangeable }\right)\end{array}$ & 466 \\
\hline $4 c$ & $\begin{array}{l}3634,3419,3047,2948,2605,1654 \\
1485,1235 .\end{array}$ & $\begin{array}{c}3.530\left(\mathrm{~s}, 1 \mathrm{H}, \mathrm{Ar}-\mathrm{SH}, \mathrm{D}_{2} \mathrm{O} \text { exchangeable), } 6.09\left(\mathrm{~s}, 1 \mathrm{H},-\mathrm{CHC}_{6} \mathrm{H}_{5}\right), 6.989(\mathrm{~s}, 1 \mathrm{H}, \mathrm{Ar}-\mathrm{H}),\right. \\
\text { 7.110-7.126 (d, } 2 \mathrm{H}, \mathrm{Ar}-\mathrm{H}), 7.136-7.173(\mathrm{t}, 3 \mathrm{H}, \mathrm{Ar}-\mathrm{H}), 7.368-7.409(\mathrm{t}, 2 \mathrm{H}, \mathrm{Ar}-\mathrm{H}), \\
\text { 7.414-7.439 (t, } 1 \mathrm{H}, \mathrm{Ar}-\mathrm{H}), 7.489-7.524(\mathrm{~d}, 2 \mathrm{H}, \mathrm{Ar}-\mathrm{H}), 7.594-7.604(\mathrm{~d}, 1 \mathrm{H}, \mathrm{Ar}-\mathrm{H}) \\
\text { 7.709-7.728 (d, } 1 \mathrm{H}, \mathrm{Ar}-\mathrm{H}), 7.784-7.799(\mathrm{~d}, 1 \mathrm{H}, \mathrm{Ar}-\mathrm{H}), 7.989-8.001(\mathrm{~d}, 1 \mathrm{H}, \mathrm{Ar}-\mathrm{H}), \\
9.099 \text { (br. s, } 1 \mathrm{H}, \mathrm{OH}, \mathrm{D}_{2} \mathrm{O} \text { exchangeable), } 10.979\left(\mathrm{~s}, 1 \mathrm{H}, \mathrm{Ar}-\mathrm{NH}, \mathrm{D}_{2} \mathrm{O} \text { exchangeable) }\right.\end{array}$ & 453 \\
\hline
\end{tabular}

Cite this article: Atul Sharma, Sanjit Kumar Das, Devender Pathak, Gyanendra Kumar Sharma. Synthesis and Pharmacological Evaluation of Tricyclic Carbazole Annulated with Substituted Benzothiazole-2-Amine Under Microwave Irradiation. Biomed J Sci\&Tech Res 9(2)-2018. BJSTR. MS.ID.001786. DOI: 10.26717/ BJSTR.2018.09.001786. 


\begin{tabular}{|c|c|c|c|}
\hline $5 c$ & $\begin{array}{c}3628,3423,3053,2958,2954,1651, \\
1491,1245 .\end{array}$ & 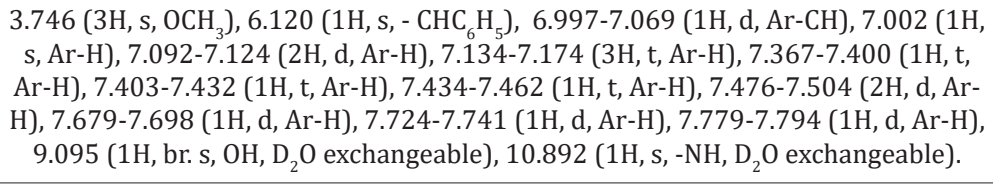 & 451 \\
\hline $6 c$ & $\begin{array}{c}3629,3415,3051,2925,1648,1482, \\
1240,1064 .\end{array}$ & $\begin{array}{c}6.111\left(1 \mathrm{H}, \mathrm{s},-\mathrm{CHC}_{6} \mathrm{H}_{5}\right), 7.014(1 \mathrm{H}, \mathrm{s}, \mathrm{Ar}-\mathrm{H}), 7.112-7.130(2 \mathrm{H}, \mathrm{d}, \mathrm{Ar}-\mathrm{H}), 7.134-7.179 \\
(3 \mathrm{H}, \mathrm{t}, \mathrm{Ar}-\mathrm{H}), 7.356-7.398(1 \mathrm{H}, \mathrm{t}, \mathrm{Ar}-\mathrm{H}), 7.367-7.400(1 \mathrm{H}, \mathrm{t}, \mathrm{Ar}-\mathrm{H}), 7.406-7.433(1 \mathrm{H}, \\
\text { t, Ar-H), 7.458-7.474 (1H, d, Ar-H), 7.491-7.506 (1H, d, Ar-H), 7.725-7.797 }(2 \mathrm{H}, \mathrm{d}, \\
\mathrm{Ar}-\mathrm{H}), 7.978-7.995(1 \mathrm{H}, \mathrm{d}, \mathrm{Ar}-\mathrm{H}), 9.052\left(1 \mathrm{H}, \text { br. s, OH, } \mathrm{D}_{2} \mathrm{O} \text { exchangeable }\right), 10.939 \\
\left(1 \mathrm{H}, \mathrm{s},-\mathrm{NH}, \mathrm{D}_{2} \mathrm{O} \text { exchangeable }\right)\end{array}$ & 489 \\
\hline $7 c$ & $\begin{array}{c}3631,3417,3049,2925,1654 \\
1491,1240,1067\end{array}$ & $\begin{array}{c}6.111\left(1 \mathrm{H}, \mathrm{s},-\mathrm{CHC}_{6} \mathrm{H}_{5}\right), 7.009(1 \mathrm{H}, \mathrm{s}, \mathrm{Ar}-\mathrm{H}), 7.112-7.129(2 \mathrm{H}, \mathrm{d}, \mathrm{Ar}-\mathrm{H}), 7.132-7.179 \\
(3 \mathrm{H}, \mathrm{t}, \mathrm{Ar}-\mathrm{H}), 7.356-7.396(1 \mathrm{H}, \mathrm{t}, \mathrm{Ar}-\mathrm{H}), 7.411-7.422(1 \mathrm{H}, \mathrm{t}, \mathrm{Ar}-\mathrm{H}), 7.453-7.474(2 \mathrm{H}, \\
\text { d, Ar-H), 7.551-7.568 (1H, d, Ar-H), 7.752-7.769 }(1 \mathrm{H}, \mathrm{d}, \mathrm{Ar}-\mathrm{H}), 7.996-8.011(2 \mathrm{H}, \mathrm{d}, \\
\mathrm{Ar}-\mathrm{H}), 8.230(1 \mathrm{H}, \mathrm{s}, \mathrm{Ar}-\mathrm{H}), 9.052(1 \mathrm{H}, \text { br. s, OH D } \mathrm{O} \text { exchangeable }), 10.943(1 \mathrm{H}, \mathrm{s}, \\
\left.-\mathrm{NH}, \mathrm{D}_{2} \mathrm{O} \text { exchangeable }\right) .\end{array}$ & 455 \\
\hline
\end{tabular}

\section{In Silico Prediction Using Pass Program}

Towards an endeavor of convergence in terms of pharmacological activity with our experimental study, the prediction of biological activity was performed by computer assisted in-silico program using PASS [21,22] a knowledge base biological activity prediction tool. The mechanism of this software is based on the SAR base of the hundred of thousand of compounds with known biological activity stored in the respective database with a diverse spectrum of chemical space. The PASS based preliminary screening of the large database with a similar and diverse structural determinant could increase the probability of finding a molecule to be active with a similar or divergent structural requirements. The PASS [23] program generally uses the two common parameters, i.e. $\mathrm{Pa}$ and $\mathrm{Pi}$ value where, $\mathrm{Pa}$ is defined by the probability of a compound being active with a certain applicability domain and the Pi is the exact opposite.

Herein, we set the [Pa] value a little higher to raise the level of constraint for a molecule to be pharmacologically active and to be sure about the consistency of the result extracted out from the program. In this prediction results, compound $2 \mathrm{~b}, 4 \mathrm{~b}$ and $5 \mathrm{~b}$ has not shown any kind of activity rather they also showed some kind of adverse effect. Additionally, on performance of adverse effect analysis, compound of similar type of molecular surface and the interaction profile showed similarities in adverse effects. The compounds 1c-7c showed the antibacterial effect possessing potassium channel blocking activity. For each and every compound in the dataset, near about 4000 types of the spectrum of activity was estimated by PASS [24,25]. A threshold for activity was chosen 0.7 and unexpectedly almost all the compound was overtaking the constraint comprehensively. The detailed result has been stipulated in the Table 3.

\section{Biological Evaluation}

In the present work, the newly synthesized derivatives (1b-7b) and $(1 \mathrm{c}-7 \mathrm{c})$ were screened via computer assisted in silico program and compared with experimentally performed biological activities. The newly synthesized compounds $1 \mathrm{c}-7 \mathrm{c}$ possess similar type of activity which predicted by PASS program as shown in Table 3 and Figure 2, the compounds $2 \mathrm{c}$ and $3 \mathrm{c}$ showed good antibacterial activity. Compounds $1 \mathrm{c}$ and $7 \mathrm{c}$ were having moderate to good activity against E. coli. and against B. subtilis. As shown in Figure 2 the compounds $2 \mathrm{c}, 3 \mathrm{c}$ and $5 \mathrm{c}$ were having good activity against $\mathrm{B}$. subtilis and $5 c$ having moderate activity against all bacterial strains. It was observed that the derivatives having electronegative substitution at C-5 and C- 6 increase the antimicrobial potential. In vitro antifungal screening of newly synthesized compounds was performed against a fifteen days old culture of $\mathrm{A}$.
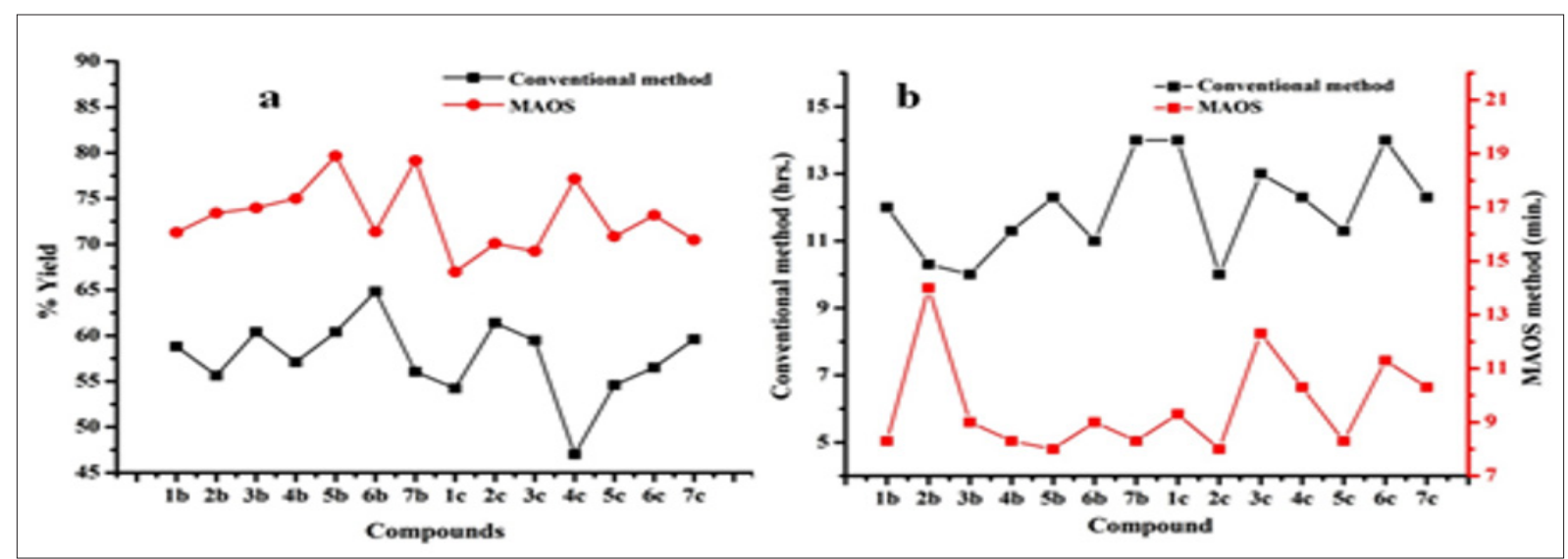

Figure 3: Comparative results of conventional and MAOS method of N-Mannich bases (1b-7b and 1c-7c) showing (a) \% yield and (b) reaction time. 
niger and C. albicans. As shown in Figure 4 the compounds $1 \mathrm{~b}$, $3 \mathrm{~b}$ and $7 \mathrm{~b}$ showed good antifungal activity against $C$. albicans and A. niger in comparison to standard drug Fluconazole at conc. $50 \mu \mathrm{g}$ $\mathrm{mL}^{-1}$. No potential of biological activity was shown by compounds $2 \mathrm{~b}, 4 \mathrm{~b}$ and $5 \mathrm{~b}$ as depicted in the Figure $3 \& 4$. However, the $2 \mathrm{~b}, 4 \mathrm{~b}$ and $5 \mathrm{~b}$ possess the severe side effects as shown in Table 3. It is note- worthy to mention here that, almost a similar type of compound series shown identical type of activity as predicted by PASS program in silico. Thus, the obtained result suggested, it is noteworthy to further explore the derivatives which showed side effects such as neutrophilic dermatosis (Sweet's syndrome) and postural (orthostatic) hypotension and no biological activity as shown in Table 3.

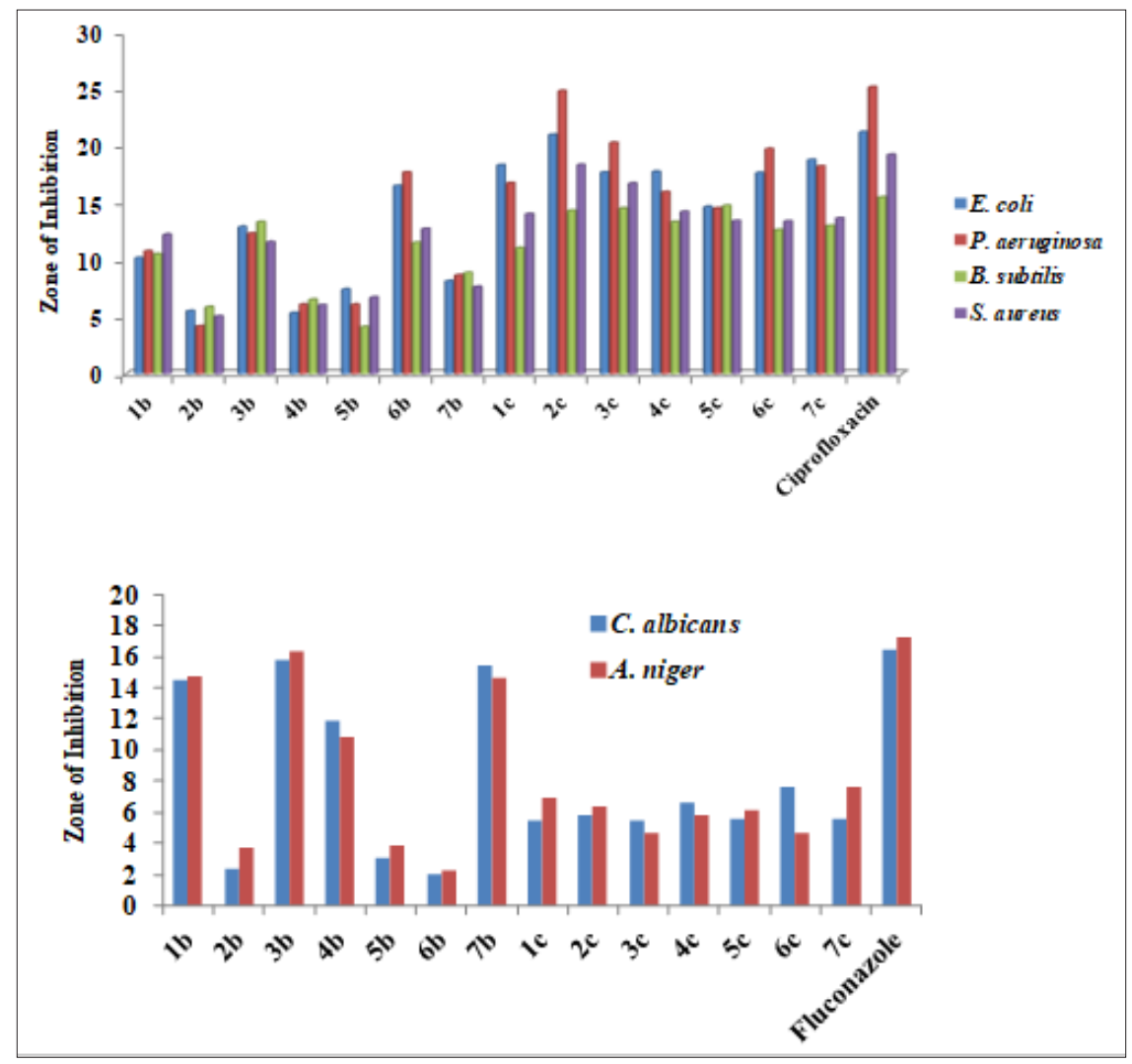

Figure 4: (a) Antibacterial activities bar graph of N-Mannich bases of carbazole; (b) Antifungal activities bar graph of N-Mannich bases of carbazole.

Table 3: Prediction results from PASS program.

\begin{tabular}{|c|c|c|c|c|c|c|c|c|c|}
\hline $\begin{array}{l}\text { Compound } \\
\text { Code }\end{array}$ & $\mathbf{P a}$ & $\mathbf{P i}$ & $\begin{array}{l}\text { Predicted mechanism of } \\
\text { action (MOA) }\end{array}$ & $\mathrm{Pa}$ & $\mathbf{P i}$ & $\begin{array}{l}\text { Predicted } \\
\text { biological } \\
\text { activity }\end{array}$ & $\mathbf{P a}$ & $\mathbf{P i}$ & Predicted adverse effect \\
\hline $1 b$ & 0.792 & 0.015 & $\begin{array}{l}\text { Glycosylphosphatidylinositol } \\
\text { phospholipase D inhibitor }\end{array}$ & 0.812 & 0.012 & Antifungal & 0.792 & 0.016 & $\begin{array}{l}\text { Postural (orthostatic) } \\
\text { hypotension }\end{array}$ \\
\hline $2 \mathrm{~b}$ & 0 & 0 & Nil & 0 & 0 & Nil & 0.752 & 0.036 & $\begin{array}{l}\text { Neutrophilic dermatosis } \\
\text { (Sweet's syndrome) }\end{array}$ \\
\hline $3 b$ & 0,716 & 0.015 & Arylacetonitrilase inhibitor & 0.712 & 0.014 & Antifungal & 0.719 & 0.046 & $\begin{array}{l}\text { Neutrophilic dermatosis } \\
\text { (Sweet's syndrome) }\end{array}$ \\
\hline $4 b$ & 0 & 0 & Nil & 0 & 0 & Nil & 0 & 0 & Nil \\
\hline $5 b$ & 0 & 0 & Nil & 0 & 0 & Nil & 0.725 & 0.025 & $\begin{array}{l}\text { Postural (orthostatic) } \\
\text { hypotension }\end{array}$ \\
\hline $6 b$ & 0.766 & 0.02 & $\begin{array}{l}\text { Glycosylphosphatidylinositol } \\
\text { phospholipase D inhibitor }\end{array}$ & 0.715 & 0.008 & Antibacterial & 0.747 & 0.072 & Twitching \\
\hline $7 b$ & 0.846 & 0.006 & $\begin{array}{l}\text { Glycosylphosphatidylinositol } \\
\text { phospholipase D inhibitor }\end{array}$ & 0.711 & 0.006 & Antifungal & 0.796 & 0.024 & $\begin{array}{l}\text { Neutrophilic dermatosis } \\
\text { (Sweet's syndrome) }\end{array}$ \\
\hline $1 \mathrm{c}$ & 0.801 & 0.003 & Potassium channel blocker & 0.814 & 0.006 & Antibacterial & 0.716 & 0.09 & Twitching \\
\hline
\end{tabular}




\begin{tabular}{|c|c|c|c|c|c|c|c|c|c|}
\hline 2c & 0.773 & 0.004 & Potassium channel blocker & 0.812 & 0.003 & Antibacterial & 0.87 & 0.007 & $\begin{array}{c}\text { Postural (orthostatic) } \\
\text { hypotension }\end{array}$ \\
\hline $3 \mathrm{c}$ & 0.777 & 0.004 & Potassium channel blocker & 0.711 & 0.012 & Antibacterial & 0.883 & 0.005 & $\begin{array}{c}\text { Postural (orthostatic) } \\
\text { hypotension }\end{array}$ \\
\hline $4 \mathrm{c}$ & 0.752 & 0.004 & Potassium channel blocker & 0.719 & 0.013 & Antibacterial & 0 & 0 & Nil \\
\hline $5 \mathrm{c}$ & 0.79 & 0.004 & Potassium channel blocker & 0.814 & 0.005 & Antibacterial & 0.749 & 0.021 & $\begin{array}{c}\text { Postural (orthostatic) } \\
\text { hypotension }\end{array}$ \\
\hline $6 \mathrm{c}$ & 0.805 & 0.003 & Potassium channel blocker & 0.786 & 0.016 & Antibacterial & 0.748 & 0.022 & $\begin{array}{c}\text { Postural (orthostatic) } \\
\text { hypotension }\end{array}$ \\
\hline $7 \mathrm{c}$ & 0.805 & 0.003 & Potassium channel blocker & 0.801 & 0.003 & Antibacterial & 0.789 & 0.047 & Twitching \\
\hline
\end{tabular}

\section{Conclusion}

In conclusion, two new series of N-Mannich bases of carbazole has been successfully synthesized. All synthesized derivatives were characterized by physicochemical and spectral analysis. The developed MAOS method revealed improved reaction condition ( $\%$ yield and reaction time) in comparison to conventional methods. Further, MAOS method can be successfully employed to increase the yield of the organic compounds. The in vitro evaluation of newly synthesized compounds revealed the potential for biological activity. Some of the derivatives showed the potent or equipotent biological potential in comparison to standard drug. In silico prediction of PASS program suggested that the similar type of compounds possess similar type of biological potential, mechanism of action and adverse effects. Prediction studies suggested that it is note essential to further explore the derivatives which only possess severe side effects no biological potential.

\section{Acknowledgment}

Atul Sharma would like to thanks to All India Council of Technical Education (AICTE), New Delhi for GATE Fellowship. Authors would like to thanks CDRI, Lucknow and Indian Institute of Technology (IIT) Delhi for instrumentation facilities.

\section{References}

1. Wang J, Zheng Y, Efferth T, Wang R, Shen Y, et al. (2005) Indole and carbazole alkaloids from Glycosmis montana with weak anti-HIV and cytotoxic activities. J Phytochem 66(6): 697-701.

2. Danish IA, Prasad KJR (2004) Syntheses and Characterization of N, $N_{B}$ biscarbazolyl azine and $\mathrm{N}, \mathrm{N}_{\mathrm{B}}$. Carbazolyl hydrazine derivatives and antimicrobial studies. Indian J Heterocycl Chem 14: 19-22.

3. Obniska J, Rzepka S, Kaminski K (2012) Synthesis and anticonvulsant activity of new N-Mannich bases derived from 3-(2-fluorophenyl)- and 3-(2-bromophenyl)-pyrrolidine-2,5-diones Part II. Bioorgan Med Chem 20(15): 4872-4880.

4. Venkatesh P, Pandeya SN (2009) Int J ChemTech Res1(4): 1354-1358.

5. Poumarox A, Bouaziz Z, Fillion H, Domard M, Giraud J, et al. (1999) Chem Pharm Bull 47 (5): 643-646.

6. Kumar R, Ramchandran U, Srinivasan K, Ramarao P, Raichur S (2005) Design, synthesis and evaluation of carbazole derivatives as PPAR $\alpha / \gamma$ dual agonists and antioxidants. Bioorg Med Chem 13(13): 4279-4290.

7. Mandal S, Nayak A, Kar M, Banerjee SK, Das A, et al. (2010) Antidiarrhoeal activity of carbazole alkaloids from Murraya koenigii Spreng (Rutaceae) seeds. Fitoterapia 81(1): 72-74.

8. Fausteris MA, Papakyriakou A, Koutsourea A, Manioudaki M, Lampropoulou E, et al. (2008) Pyrrolo [2,3-a] carbazoles as Potential Cyclin Dependent Kinase 1 (CDK1) Inhibitors. Synthesis, Biological Evaluation, and Binding Mode through Docking Simulations. J Med Chem 51(4): 1048-1052.
9. Garg LC, Atal CK (1969) Ind J Pharmacol 32: 104.

10. Haider N, Marian B, Nagel T, Tarnaia M, Troppera K (2014) Electrophilic Substitution of Dimethyl 1-Methylcarbazole-2,3-dicarboxylate: Synthesis of New b-Fused Carbazoles as Potential Antitumor Agents. J Braz Chem Soc 25 (11): 1965-1974.

11. Pierce LT, Cahill MM, Winfield HJ, McCarthy, FO (2012) Synthesis and identification of novel indolo [2,3-a] pyrimido [5,4-c] carbazoles as a new class of anti-cancer agents. Eur J Med Chem 56: 292-300.

12. Ames BN (1983) Dietary carcinogens and anticarcinogens. Oxygen radicals and degenerative diseases. Science 221(4617): 1256-1264.

13. Rajendran A, Ramu S, Karthikeyan C (2011) Int J Curr Res 33 (2): 161167.

14. Zhang FF, Gan LL, Zhou CH (2010) Synthesis, antibacterial and antifungal activities of some carbazole derivatives. Bioorg Med Chem Lett 20(6): 1881-1884.

15. Bamnela R, Shrivastava SP (2012) Chem Sci Trans 1(2): 431-439.

16. Rajamanickam V, Rajasekarena A, Palanivelu M, Anandarajagopal K, Elahi ALA, et al. (2008) Int J Chem Sci 6(3): 1669-1675.

17. Bekircan O, Bektas H (2008) Synthesis of Schiff and Mannich Bases of Isatin Derivatives with 4-Amino-4,5-Dihydro-1H-1,2,4-Triazole-5-Ones. Molecules 13(9): 2126-2135.

18. Aanandhi MV, Verma AK, Sujatha R, Raj RK (2013) synthesis and characterization of novel mannich bases of benzimidazole derivatives for antibacterial and antifungal activity. Int J Pharma Pharmaceut Sci $5(2)$.

19. Barbieri V, Ferlin MG (2006) Tetrahedron letters 47: 8289-8292.

20. Stepanchikova AV, Lagunin AA, Filimonov DA, Poroikov VV (2003) Prediction of biological activity spectra for substances: evaluation on the diverse sets of drug-like structures. Curr Med Chem 10(3): 225-233.

21. Proikov VV, Filimonov DA (2002) How to acquire new biological activities in old compounds by computer prediction. J Comput Aided Mol Des 16(11): 819-824.

22. Proikov VV, Filimonov D, Lagunin A, Gloriozova T, Zakharov A (2007) PASS: identification of probable targets and mechanisms of toxicity. SAR QSAR Environ Res 18(1-2): 101-110.

23. Geronikaki A, Druzhikovsky D, Zakharov A, Proikov V (2008) Computeraided prediction for medicinal chemistry via the Internet1. SAR QSAR Environ Res 19(1,2): 27-38.

24. Dhiman A, Nanda A, Ahmad S, Narasimhan B (2011) In vitro antimicrobial activity of methanolic leaf extract of Psidium guajava L. J Pharm Bioallied Sci 3(2): 226-229.

25. Martin AE, Prasad KJR (2006) Synthesis and characterization of carbazole derivatives and their antimicrobial studies. Acta Pharm 56(1): 77-86.

26. Schrodinger small molecule drug discovery suite, Maestro molecular visualization program. 


\section{ISSN: 2574-1241}

DOI: 10.26717/BJSTR.2018.09.001786

Atul Sharma, Gyanendra Kumar S. Biomed J Sci \& Tech Res

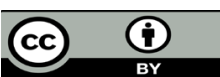

This work is licensed under Creative Commons Attribution 4.0 License

Submission Link: https://biomedres.us/submit-manuscript.php

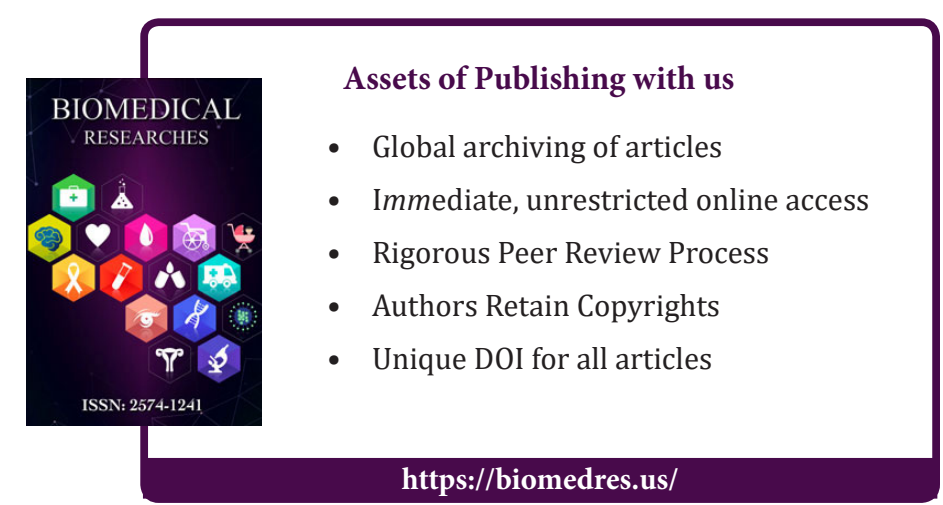

